BULLETIN (New Series) OF THE

AMERICAN MATHEMATICAL SOCIETY

Volume 37, Number 1, Pages 55-64

S 0273-0979(99)00802-2

Article electronically published on December 21, 1999

\title{
INTUITIONISM AND FORMALISM
}

\author{
DR. L. E. J. BROUWER
}

The subject for which I am asking your attention deals with the foundations of mathematics. To understand the development of the opposing theories existing in this field one must first gain a clear understanding of the concept "science"; for it is as a part of science that mathematics originally took its place in human thought.

By science we mean the systematic cataloguing by means of laws of nature of causal sequences of phenomena, i. e., sequences of phenomena which for individual or social purposes it is convenient to consider as repeating themselves identically, and more particularly of such causal sequences as are of importance in social relations.

That science lends such great power to man in his action upon nature is due to the fact that the steadily improving cataloguing of ever more causal sequences of phenomena gives greater and greater possibility of bringing about desired phenomena, difficult or impossible to evoke directly, by evoking other phenomena connected with the first by causal sequences. And that man always and everywhere creates order in nature is due to the fact that he not only isolates the causal sequences of phenomena (i. e., he strives to keep them free from disturbing secondary phenomena) but also supplements them with phenomena caused by his own activity, thus making them of wider applicability. Among the latter phenomena the results of counting and measuring take so important a place, that a large number of the natural laws introduced by science treat only of the mutual relations between the results of counting and measuring. It is well to notice in this connection that a natural law in the statement of which measurable magnitudes occur can only be understood to hold in nature with a certain degree of approximation; indeed natural laws as a rule are not proof against sufficient refinement of the measuring tools.

The exceptions to this rule have from ancient times been practical arithmetic and geometry on the one hand, and the dynamics of rigid bodies and celestial mechanics on the other hand. Both these groups have so far resisted all improvements in the tools of observation. But while this has usually been looked upon as something accidental and temporal for the latter group, and while one has always been prepared to see these sciences descend to the rank of approximate theories, until comparatively recent times there has been absolute confidence that no experiment could ever disturb the exactness of the laws of arithmetic and geometry; this confidence is expressed in the statement that mathematics is "the" exact science.

On what grounds the conviction of the unassailable exactness of mathematical laws is based has for centuries been an object of philosophical research, and two

2000 Mathematics Subject Classification. Primary 03-03, 01-02, 03Fxx.

Reprinted from Bull. Amer. Math. Soc. 20 (1913), 81-96.

Translated for the Bulletin by Professor ARnold Dresden.

Inaugural address at the University of Amsterdam, read October 14, 1912.

(C)1999 American Mathematical Society 
points of view may here be distinguished, intuitionism (largely French) and formalism (largely German). In many respects these two viewpoints have become more and more definitely opposed to each other; but during recent years they have reached agreement as to this, that the exact validity of mathematical laws as laws of nature is out of the question. The question where mathematical exactness does exist, is answered differently by the two sides; the intuitionist says: in the human intellect, the formalist says: on paper.

In Kant we find an old form of intuitionism, now almost completely abandoned, in which time and space are taken to be forms of conception inherent in human reason. For Kant the axioms of arithmetic and geometry were synthetic a priori judgments, i. e., judgments independent of experience and not capable of analytical demonstration; and this explained their apodictic exactness in the world of experience as well as in abstracto. For Kant, therefore, the possibility of disproving arithmetical and geometrical laws experimentally was not only excluded by a firm belief, but it was entirely unthinkable.

Diametrically opposed to this is the view of formalism, which maintains that human reason does not have at its disposal exact images either of straight lines or of numbers larger than ten, for example, and that therefore these mathematical entities do not have existence in our conception of nature any more than in nature itself. It is true that from certain relations among mathematical entities, which we assume as axioms, we deduce other relations according to fixed laws, in the conviction that in this way we derive truths from truths by logical reasoning, but this non-mathematical conviction of truth or legitimacy has no exactness whatever and is nothing but a vague sensation of delight arising from the knowledge of the efficacy of the projection into nature of these relations and laws of reasoning. For the formalist therefore mathematical exactness consists merely in the method of developing the series of relations, and is independent of the significance one might want to give to the relations or the entities which they relate. And for the consistent formalist these meaningless series of relations to which mathematics are reduced have mathematical existence only when they have been represented in spoken or written language together with the mathematical-logical laws upon which their development depends, thus forming what is called symbolic logic.

Because the usual spoken or written languages do not in the least satisfy the requirements of consistency demanded of this symbolic logic, formalists try to avoid the use of ordinary language in mathematics. How far this may be carried is shown by the modern Italian school of formalists, whose leader, Peano, published one of his most important discoveries concerning the existence of integrals of real differential equations in the Mathematische Annalen in the language of symbolic logic; the result was that it could only be read by a few of the initiated and that it did not become generally available until one of these had translated the article into German.

The viewpoint of the formalist must lead to the conviction that if other symbolic formulas should be substituted for the ones that now represent the fundamental mathematical relations and the mathematical-logical laws, the absence of the sensation of delight, called "consciousness of legitimacy," which might be the result of such substitution would not in the least invalidate its mathematical exactness. To the philosopher or to the anthropologist, but not to the mathematician, belongs the task of investigating why certain systems of symbolic logic rather than others may be effectively projected upon nature. Not to the mathematician, but to the psychologist, belongs the task of explaining why we believe in certain systems of symbolic 
logic and not in others, in particular why we are averse to the so-called contradictory systems in which the negative as well as the positive of certain propositions are valid. ${ }^{1}$

As long as the intuitionists adhered to the theory of Kant it seemed that the development of mathematics in the nineteenth century put them in an ever weaker position with regard to the formalists. For in the first place this development showed repeatedly how complete theories could be carried over from one domain of mathematics to another: projective geometry, for example, remained unchanged under the interchange of the rôles of point and straight line, an important part of the arithmetic of real numbers remained valid for various complex number fields and nearly all the theorems of elementary geometry remained true for non-archimedian geometry, in which there exists for every straight line segment another such segment, infinitesimal with respect to the first. These discoveries seemed to indicate indeed that of a mathematical theory only the logical form was of importance and that one need no more be concerned with the material than it is necessary to think of the significance of the digit groups with which one operates, for the correct solution of a problem in arithmetic.

But the most serious blow for the Kantian theory was the discovery of noneuclidean geometry, a consistent theory developed from a set of axioms differing from that of elementary geometry only in this respect that the parallel axiom was replaced by its negative. For this showed that the phenomena usually described in the language of elementary geometry may be described with equal exactness, though frequently less compactly in the language of non-euclidean geometry; hence it is not only impossible to hold that the space of our experience has the properties of elementary geometry but it has no significance to ask for the geometry which would be true for the space of our experience. It is true that elementary geometry is better suited than any other to the description of the laws of kinematics of rigid bodies and hence of a large number of natural phenomena, but with some patience it would be possible to make objects for which the kinematics would be more easily interpretable in terms of non-euclidean than in terms of euclidean geometry. ${ }^{2}$

However weak the position of intuitionism seemed to be after this period of mathematical development, it has recovered by abandoning Kant's apriority of space but adhering the more resolutely to the apriority of time. This neo-intuitionism considers the falling apart of moments of life into qualitatively different parts, to be reunited only while remaining separated by time as the fundamental phenomenon of the human intellect, passing by abstracting from its emotional content into the fundamental phenomenon of mathematical thinking, the intuition of the bare twooneness. This intuition of two-oneness, the basal intuition of mathematics, creates not only the numbers one and two, but also all finite ordinal numbers, inasmuch as one of the elements of the two-oneness may be thought of as a new two-oneness, which process may be repeated indefinitely; this gives rise still further to the smallest infinite ordinal number $\omega$. Finally this basal intuition of mathematics, in which the connected and the separate, the continuous and the discrete are united, gives rise immediately to the intuition of the linear continuum, i. e., of the "between," which is not exhaustible by the interposition of new units and which therefore can never be thought of as a mere collection of units.

\footnotetext{
${ }^{1}$ See Mannoury, "Methodologisches und Philosophisches zur Elementarmathematik," pp. 149154.

${ }^{2}$ See Poincaré, La Science et l'Hypothèse, p. 104.
} 
In this way the apriority of time does not only qualify the properties of arithmetic as synthetic a priori judgments, but it does the same for those of geometry, and not only for elementary two- and three-dimensional geometry, but for non-euclidean and $n$-dimensional geometries as well. For since Descates we have learned to reduce all these geometries to arithmetic by means of the calculus of coordinates.

From the present point of view of intuitionism therefore all mathematical sets of units which are entitled to the name can be developed out of the basal intuition, and this can only be done by combining a finite number of times the two operations: "to create a finite ordinal number" and "to create the infinite ordinal number $\omega "$; here it is to be understood that for the latter purpose any previously constructed set or any previously performed constructive operation may be taken as a unit. Consequently the intuitionist recognizes only the existence of denumerable sets, i. e., sets whose elements may be brought into one-to-one correspondence either with the elements of a finite ordinal number or with those of the infinite ordinal number $\omega$. And in the construction of these sets neither the ordinary language nor any symbolic language can have any other rôle than that of serving as a nonmathematical auxiliary, to assist the mathematical memory or to enable different individuals to build up the same set.

For this reason the intuitionist can never feel assured of the exactness of a mathematical theory by such guarantees as the proof of its being non-contradictory, ${ }^{3}$ the possibility of defining its concepts by a finite number of words, ${ }^{3}$ or the practical certainty that it will never lead to a misunderstanding in human relations. ${ }^{4}$

As has been stated above, the formalist wishes to leave to the psychologist the task of selecting the "truly-mathematical" language from among the many symbolic languages that may be consistently developed. Inasmuch as psychology has not yet begun on this task, formalism is compelled to mark off, at least temporarily, the domain that it wishes to consider as "true mathematics" and to lay down for that purpose a definite system of axioms and laws of reasoning, if it does not wish to see its work doomed to sterility. The various ways in which this attempt has actually been made all follow the same leading idea, viz., the presupposition of the existence of a world of mathematical objects, a world independent of the thinking individual, obeying the laws of classical logic and whose objects may possess with respect to each other the "relation of a set to its elements." With reference to this relation various axioms are postulated, suggested by the practice with natural finite sets; the principal of these are: "a set is determined by its elements"; "for any two mathematical objects it is decided whether or not one of them is contained in the other one as an element"; "to every set belongs another set containing as its elements nothing but the subsets of the given set"; the axiom of selection: " $a$ set which is split into subsets contains at least one subset which contains one and not more than one element of each of the first subsets; the axiom of inclusion: "if for any mathematical object it is decided whether a certain property is valid for it or not, then there exists a set containing nothing but those objects for which the property does hold"; the axiom of composition: "the elements of all sets that belong to a set of sets form a new set."

On the basis of such a set of axioms the formalist develops now in the first place the theory of "finite sets." A set is called finite if its elements can not be

\footnotetext{
${ }^{3}$ See however Poincaré in Scientia, No. XXIV, p. 6.

${ }^{4}$ See however Borel in Revue du Mois, No. 80, p. 221.
} 
brought into one-to-one correspondence with the elements of one of its subsets; by means of relatively complicated reasoning the principle of complete induction is proved to be a fundamental property of these sets; ${ }^{5}$ this principle states that a property will be true for all finite sets if, first, it is true for all sets containing a single element, and, second, its validity for an arbitrary finite set follows from its validity for this same set reduced by a single one of its elements. That the formalist must give an explicit proof of this principle, which is self-evident for the finite numbers of the intuitionist on account of their construction, shows at the same time that the former will never be able to justify his choice of axioms by replacing the unsatisfactory appeal to inexact practice or to intuition equally inexact for him by a proof of the non-contradictoriness of his theory. For in order to prove that a contradiction can never arise among the infinitude of conclusions that can be drawn from the axioms he is using, he would first have to show that if no contradiction had as yet arisen with the $n$th conclusion then none could arise with the $(n+1)$ th conclusion, and secondly he would have to apply the principle of complete induction intuitively. But it is this last step which the formalist may not take, even though he should have proved the principle of complete induction; for this would require mathematical certainty that the set of properties obtained after the $n$th conclusion had been reached, would satisfy for an arbitrary $n$ his definition for finite sets, ${ }^{6}$ and in order to secure this certainly he would have to have recourse not only to the unpermissible application of a symbolic criterion to a concrete example but also to another intuitive application of the principle of complete induction; this would lead him to a vicious circle reasoning.

In the domain of finite sets in which the formalistic axioms have an interpretation perfectly clear to the intuitionists, unreservedly agreed to by them, the two tendencies differ solely in their method, not in their results; this becomes quite different however in the domain of infinite or transfinite sets, where, mainly by the application of the axiom of inclusion, quoted above, the formalist introduces various concepts, entirely meaningless to the intuitionist, such as for instance "the set whose elements are the points of space," "the set whose elements are the continuous functions of a variable," "the set whose elements are the discontinuous functions of a variable," and so forth. In the course of these formalistic developments it turns out that the consistent application of the axiom of inclusion leads inevitably to contradictions. A clear illustration of this fact is furnished by the so-called paradox of Burali-Forti. ${ }^{7}$ To exhibit it we have to lay down a few definitions.

A set is called ordered if there exists between any two of its elements a relation of "higher than" or "lower than," with this understanding that if the element $a$ is higher than the element $b$, then the element $b$ is lower than the element $a$, and if the element $b$ is higher than $a$ and $c$ is higher than $b$, then $c$ is higher than $a$.

A well-ordered set (in the formalistic sense) is an ordered set, such that every subset contains an element lower than all others.

Two well-ordered sets that may be brought into one-to-one correspondence under invariance of the relations of "higher than" and "lower than" are said to have the same ordinal number.

\footnotetext{
${ }^{5}$ Compare, e. g., Zermelo, "Sur les ensembles finis et le principe de l'induction complète," Acta Mathematica, 32, pp. 185-193.

${ }^{6}$ Compare Poincaré, Revue de Métaphysique et de Morale, 1905, p. 834.

${ }^{7}$ Compare Rendiconti del Circolo Matematico di Palermo, 1897.
} 
If two ordinal numbers $A$ and $B$ are not equal, then one of them is greater than the other one, let us say $A$ is greater than $B$; this means that $B$ may be brought into one-to-one correspondence with an initial segment of $A$ under invariance of the relations of "higher than" and "lower than." We have introduced above, from the intuitionist viewpoint, the smallest infinite ordinal number $\omega$, i. e., the ordinal number of the set of all finite ordinal numbers arranged in order of magnitude. ${ }^{8}$ Well-ordered sets having the ordinal number $\omega$ are called elementary series.

It is proved without difficulty by the formalist that an arbitrary subset of a wellordered set is also a well-ordered set, whose ordinal number is less than or equal to that of the original set; also, that if to a well-ordered set that does not contain all mathematical objects a new element be added that is defined to be higher than all elements of the original set, a new well-ordered set arises whose ordinal number is greater than that of the first set.

We construct now on the basis of the axiom of inclusion the set $s$ which contains as elements all the ordinal numbers arranged in order of magnitude; then we can prove without difficulty, on the one hand that $s$ is a well-ordered set whose ordinal number can not be exceeded by any other ordinal number in magnitude, and on the other hand that it is possible, since not all mathematical objects are ordinal numbers, to create an ordinal number greater than that of $s$ by adding a new element to $s$, a contradiction. ${ }^{9}$

Although the formalists must admit contradictory results as mathematical if they want to be consistent, there is something disagreeable for them in a paradox like that of Burali-Forti because at the same time the progress of their arguments is guided by the principium contradictionis, i. e., by the rejection of the simultaneous validity of two contradictory properties. For this reason the axiom of inclusion has been modified to read as follows: "If for all elements of a set it is decided whether a certain property is valid for them or not, then the set contains a subset containing nothing but those elements for which the property does hold." 10

In this form the axiom permits only the introduction of such sets as are subsets of sets previously introduced; if one wishes to operate with other sets, their existence must be explicitly postulated. Since however in order to accomplish anything at all the existence of a certain collection of sets will have to be postulated at the outset, the only valid argument that can be brought against the introduction of a new set is that it leads to contradictions; indeed the only modifications that the discovery of paradoxes has brought about in the practice of formalism has been the abolition of those sets that had given rise to these paradoxes. One continues to operate without hesitation with other sets introduced on the basis of the old axiom of inclusion; the result of this is that extended fields of research, which are without significance for the intuitionist are still of considerable interest to the formalist. An example of this

\footnotetext{
${ }^{8}$ The more general ordinal numbers of the intuitionist are the numbers constructed by means of Cantor's two principles of generation (compare Math. Annalen, vol. 49, p. 226).

${ }^{9}$ It is without justice that the paradox of Burali-Forti is sometimes classed with the of Richard, which in a somewhat simplified form reads as follows: "Does there exist a least integer, that can not be defined by a sentence of at most twenty words? On the one hand yes, for the number of sentences of at most twenty words is of course finite; on the other hand no, for if it should exist, it would be defined by the sentence of fifteen words formed by the words italicized above."

The origin of this paradox does not lie in the axiom of inclusion but in the variable meaning of the word "defined" in the italicized sentence, which makes it possible to define by means of this sentence an infinite number of integers in succession.

${ }^{10}$ Compare Zermelo, Math. Annalen, vol. 65, p. 263.
} 
is found in the theory of potencies, of which I shall sketch the principal features here, because it illustrates so clearly the impassable chasm which separates the two sides.

Two sets are said to possess the same potency, or power, if their elements can be brought into one-to-one correspondence. The power of set $A$ is said to be greater than that of $B$, and the power of $B$ less than that of $A$, if it is possible to establish a one-to-one correspondence between $B$ and a part of $A$, but impossible to establish such a correspondence between $A$ and a part of $B$. The power of a set which has the same power as one of its subsets, is called infinite, other powers are called finite. Sets that have the same power as the ordinal number $\omega$ are called denumerably infinite and the power of such sets is called aleph-null: it proves to be the smallest infinite power. According to the statements previously made, this power aleph-null is the only infinite power of which the intuitionists recognize the existence.

Let us now consider the concept: "denumerably infinite ordinal number." From the fact that this concept has a clear and well-defined meaning for both formalist and intuitionist, the former infers the right to create the "set of all denumerably infinite ordinal numbers," the power of which he calls aleph-one, a right not recognized by the intuitionist. Because it is possible to argue to the satisfaction of both formalist and intuitionist, first, that denumerably infinite sets of denumerably infinite ordinal numbers can be built up in various ways, and second, that for every such set it is possible to assign a denumerably infinite ordinal number, not belonging to this set, the formalist concludes: "aleph-one is greater than aleph-null," a proposition, that has no meaning for the intuitionist. Because it is possible to argue to the satisfaction of both formalist and intuitionist that it is impossible to construct $^{11}$ a set of denumerably infinite ordinary numbers, which could be proved to have a power less than that of aleph-one, but greater than that of aleph-null, the formalist concludes: "aleph-one is the second smallest infinite ordinal number," a proposition that has no meaning for the intuitionist.

Let us consider the concept: "real number between 0 and 1." For the formalist this concept is equivalent to "elementary series of digits after the decimal point," ${ }^{12}$ for the intuitionist it means "law for the construction of an elementary series of digits after the decimal point, built up by means of a finite number of operations." And when the formalist creates the "set of all real numbers between 0 and 1," these words are without meaning for the intuitionist, even whether one thinks of the real numbers of the formalist, determined by elementary series of freely selected digits, or of the real numbers of the intuitionist, determined by elementary series of freely selected digits, or of the real numbers of the intuitionist, determined by finite laws of construction. Because it is possible to prove to the satisfaction of both formalist and intuitionist, first, that denumerably infinite sets of real numbers between 0 and 1 can be constructed in various ways, and second that for every such set it is possible to assign a real number between 0 and 1 , not belonging to the set, the formalist concludes: "the power of the continuum, i. e., the power of the set of real numbers between 0 and 1, is greater than aleph-null," a proposition which

\footnotetext{
${ }^{11}$ If "construct" were here replaced by "define" (in the formalistic sense), the proof would not be satisfactory to the intuitionist. For, in Cantor's argument in Math. Annalen, vol. 49, it is not allowed to replace the words "können wir bestimmen" (p. 214, line 17 from top) by the words "muss es geben."

${ }^{12}$ Here as everywhere else in this paper, the assumption is tacitly made that there are an infinite number of digits different from 9 .
} 
is without meaning for the intuitionist; the formalist further raises the question, whether there exist sets of real numbers between 0 and 1 , whose power is less than that of the continuum, but greater than aleph-null, in other words, "whether the power of the continuum is the second smallest infinite power," and this question, which is still waiting for an answer, he considers to be one of the most difficult and most fundamental of mathematical problems.

For the intuitionist, however, the question as stated is without meaning; and as soon as it has been so interpreted as to get a meaning, it can easily be answered.

If we restate the question in this form: "Is it impossible to construct ${ }^{13}$ infinite sets of real numbers between 0 and 1 , whose power is less than that of the continuum, but greater than aleph-null?," then the answer must be in the affirmative; for the intuitionist can only construct denumerable sets of mathematical objects and if, on the basis of the intuition of the linear continuum, he admits elementary series of free selections as elements of construction, then each non-denumerable set constructed by means of it contains a subset of the power of the continuum.

If we restate the question in the form: "Is it possible to establish a one-toone correspondence between the elements of a set of denumerably infinite ordinal numbers on the one hand, and a set of real numbers between 0 and 1 on the other hand, both sets being indefinitely extended by the construction of new elements, of such a character that the correspondence shall not be disturbed by any continuation of the construction of both sets?," then the answer must also be in the affirmative, for the extension of both sets can be divided into phases in such a way as to add a denumerably infinite number of elements during each phase. ${ }^{14}$

If however we put the question in the following form: "Is it possible to construct a law which will assign a denumerably infinite ordinal number to every elementary series of digits and which will give certainty a priori that two different elementary series will never have the same denumerably infinite ordinal number corresponding to them?," then the answer must be in the negative; for this law of correspondence must prescribe in some way a construction of certain denumerably infinite ordinal numbers at each of the successive places of the elementary series; hence there is for each place $c_{\nu}$ a well-defined largest denumerably infinite number $\alpha_{\nu}$, the construction of which is suggested by that particular place; there is then also a well-defined denumerably infinite ordinal number $\alpha_{\omega}$, greater than all $\alpha_{\nu}$ 's and that can not therefore be exceeded by any of the ordinal numbers involved by the law of correspondence; hence the power of that set of ordinal numbers can not exceed aleph-null.

As a means for obtaining ever greater powers, the formalists define with every power $\mu$ a "set of all the different ways in which a number of selections of power $\mu$ may be made," and they prove that the power of this set is greater that $\mu$.

\footnotetext{
${ }^{13}$ If "construct" were here replaced by "define" (in the formalistic sense), and if we suppose that the problem concerning the pairs of digits in the decimal fraction development of $\pi$, discussed on p. 95, can not be solved, then the question of the text must be answered negatively. For, let us denote by $Z$ the set of those infinite binary fractions, whose $n$th digit is 1 , if the $n$th pair of digits in the decimal fraction development of $\pi$ consists of unequal digits; let us further denote by $X$ the set of all finite binary fractions. Then the power of $Z+X$ is greater than aleph-null, but less than that of the continuum.

${ }^{14}$ Calling denumerably unfinished all sets of which the elements can be individually realized, and in which for every denumerably infinite subset there exists an element not belonging to this subset, we can say in general, in accordinance with the definitions of the text: "All denumerably unfinished sets have the same power."
} 
In particular, when it has been proved to the satisfaction of both formalist and intuitionist that it is possible in various ways to construct laws according to which functions of a real variable different from each other are made to correspond to all elementary series of digits, but that it is impossible to construct a law according to which an elementary series of digits is made to correspond to every function of a real variable and in which there is certainty a priori that two different functions will never have the same elementary series corresponding to them, the formalist concludes: "the power $c^{\prime}$ of the set of all functions of a real variable is greater than the power $c$ of the continuum," a proposition without meaning to the intuitionist; and in the same way in which he was led from $c$ to $c^{\prime}$, he comes from $c^{\prime}$ to a still greater power $c^{\prime \prime}$.

A second method used by the formalists for obtaining ever greater powers is to define for every power $\mu$, which can serve as a power of ordinary numbers, "the set of all ordinal numbers of power $\mu$," and then to prove that the power of this set is greater than $\mu$. In particular they denote by aleph-two the power of the set of all ordinal numbers of power aleph-one and they prove that aleph-two is greater than aleph-one and that it follows in magnitude immediately after aleph-one. If it should be possible to interpret this result in a way in which it would have meaning for the intuitionist, such interpretation would not be as simple in this case as it was in the preceding cases.

What has been treated so far must be considered to be the negative part of the theory of potencies; for the formalist there also exists a positive part however, founded on the theorem of Bernstein: "If the set $A$ has the same power as a subset of $B$ and $B$ has the same power as a subset of $A$, then $A$ and $B$ have the same power" or, in an equivalent form: "If the set $A=A_{1}+B_{1}+C_{1}$, has the same power as the set $A_{1}$, then it also has the same power as the set $A_{1}+B_{1}$.

This theorem is self-evident for denumerable sets. If it is to have any meaning at all for sets of higher power for the intuitionist, it will have to be interpretable as follows: "If it is possible, first to construct a law determining a one-to-one correspondence between the mathematical entities of type $A$ and those of type $A_{1}$ and second to construct a law determining a one-to-one correspondence between the mathematical entities of type $A$ and those of types $A_{1}, B_{1}$ and $C_{1}$, then it is possible to determine from these two laws by means of a finite number of operations a third law, determining a one-to-one correspondence between the mathematical entities of type $A$ and those of types $A_{1}$ and $B_{1}$."

In order to investigate the validity of this interpretation, we quote the proof:

"From the division of $A$ in to $A_{1}+B_{1}+C_{1}$, we secure by means of the correspondence $\gamma_{1}$ between $A$ and $A_{1}$ a division of $A_{1}$ into $A_{2}+B_{2}+C_{2}$, as well as a one-to-one correspondence $\gamma_{2}$ between $A_{1}$ and $A_{2}$. From the division of $A_{1}$ into $A_{2}+B_{2}+C_{2}$, we secure by means of the correspondence between $A_{1}$ and $A_{2}$ a division of $A_{2}$ into $A_{3}+B_{3}+C_{3}$, as well as a one-to-one correspondence $\gamma_{3}$ between $A_{2}$ and $A_{3}$. Indefinite repetition of this procedure will divide the set $A$ into an elementary series of subsets $C_{1}, C_{2}, C_{3}, \ldots$, an elementary series of subsets $B_{1}, B_{2}, B_{3} \ldots$, and a remainder set $D$. The correspondence $\gamma_{C}$ between $A$ and $A_{1}+B_{1}$ which is desired is secured by assigning to every element of $C_{\nu}$ the corresponding element of $C_{\nu+1}$ and by assigning every other element of $A$ to itself."

In order to test this proof on a definite example, let us take for $A$ the set of all real numbers between 0 and 1 , represented by infinite decimal fractions, for $A_{1}$ the set of those decimal fractions in which the $(2 n-1)$ th digit is equal to the $2 n$th digit; 
further a decimal fraction that does not belong to $A_{1}$ will be counted to belong to $B_{1}$ or to $C_{1}$, according as the above-mentioned equality of digits occurs an infinite or a finite number of times. By replacing successively each digit of an arbitrary element of $A$ by a pair of digits equal to it, we secure at once a law determining a one-to-one correspondence $\gamma_{1}$ between $A$ and $A_{1}$. For the element of $A_{1}$ that corresponds to an arbitrary well-defined element of $A$, such as, e. g., $\pi-3$, we can determine successively as many digits as we please; it must therefore be considered as being well-defined.

In order to determine the element corresponding to $\pi-3$ according to the correspondence $\gamma_{C}$, it is now necessary to decide first whether it happens an infinite or a finite number of times in the decimal fraction development of $\pi-3$ that a digit in an odd-numbered place is equal to the digit in the following even-numbered place; for this purpose we should either have to invent a process for constructing an elementary series of such pairs of equal digits, or to deduce a contradiction from the assumption of the existence of such an elementary series. There is, however, no ground for believing that either of these problems can be solved.

Hence it has become evident that also the theorem of Bernstein, and with it the positive part of the theory of potencies, does not allow an intuitionistic interpretation.

So far my exposition of the fundamental issue, which divides the mathematical world. There are eminent scholars on both sides and the chance of reaching an agreement within a finite period is practically excluded. To speak with Poincaré: "Les hommes ne s'entendent pas, parce qu'ils ne parlent pas la même langue et qu'il y a des langues qui ne s'apprennent pas." 